\title{
Implementation of Automatic Differentiation tools for Multicriteria IMRT Optimization
}

\section{INTRODUCTION}

Intensity Modulated Radiation Therapy (IMRT) is one of the most technically advanced treatment methods in external beam radiation therapy. Unlike conventional methods, IMRT deliver radiation beams that are effectively broken into hundreds of pieces (i.e., beamlets) with each having different intensities. The ability to manipulate the intensities of individual beamlets permits a greatly increased control over radiation fluence that enters the patient body. Therefore, when optimal patterns of beamlet intensities are chosen and used, IMRT can typically result in higher tumor control and decreased toxicity to normal tissues. Factors that affect the optimal results are determined based on the radiation dose to the tissues and its biological effects. Deviations of these factors from optimal values constitute IMRT optimization problems (in a variety of mathematical forms) and therefore minimized during the optimization process.

IMRT optimization problems are inherently multi-objective, involving many structures and their corresponding objectives. Our standard practice of solving such multi-objective problems has been a weighted-sum approach, in which a single objective function is formulated using original objectives and weights, and conventional optimization algorithms are used to minimize the single function value. Although this approach provides a straightforward means of simplifying the complex multi-objective problem, it is not always trivial to come up with a right set of weights that properly represents clinicians' preferences on individual objectives. In order to improve the process of articulating such preferences, a multicriteria strategy called Lexicographic approach (also known as preemptive approach in goal programming) has been tested for various IMRT cases.

\section{METHODS}

The lexicographic method [1] involves solving multiple optimization problems in sequence, rather than minimizing a single function once. The first step in this method is to categorize objective functions into different levels in order of importance from one to $k$, where $k$ is the index for the final level. Then the following problems are solved one after another in order of importance while keeping optimized results, $F_{j}\left(\mathbf{x}_{j}^{*}\right)$, constrained for the successive problems:

Find $\mathbf{x} \in \mathbf{R}^{n}$ such that

$$
\operatorname{Min} F_{i}(\mathbf{x})
$$

subject to

$$
F_{j}(\mathbf{x}) \leq F_{j}\left(\mathbf{x}_{j}^{*}\right)
$$

where $j=1,2, \ldots, i-1 ; i>1$ and $i=1,2, \ldots, k$

As the method proceeds, the number of constraints grows up to $k-1$ until an optimal solution $\mathbf{x}$ is found.

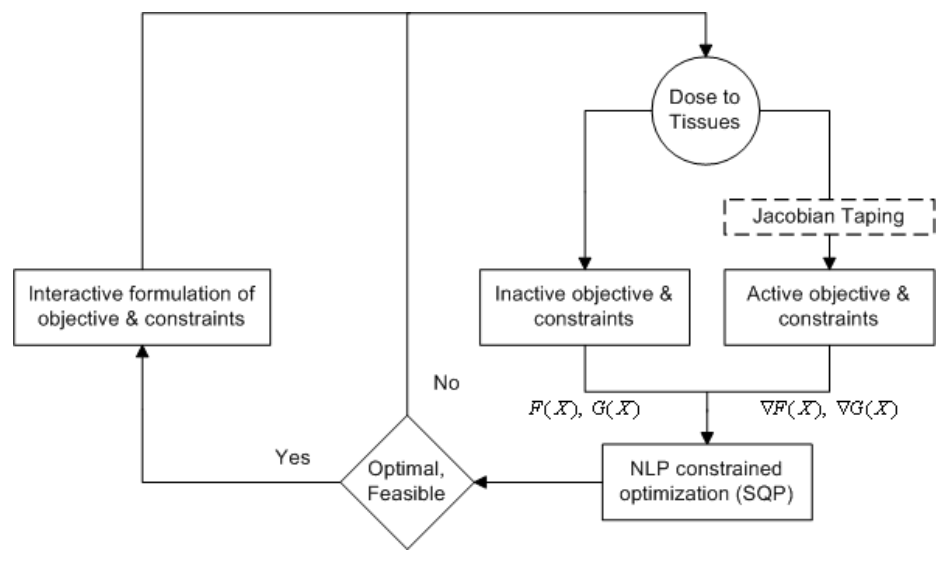

As illustrated in the diagram above, at each level of optimization, the objective and the constraints are interactively formulated by a planner using a graphical user interface (developed with Advanced Visual Systems libraries). Each optimization problem is solved by a reduced-Hessian sequential quadratic programming method (SNOPT [2]). The SQP method is expected to show particularly good performance since IMRT problems often involve significant non-linearities and a large number of optimization variables (e.g., thousands of beamlets). For the very same reason, Jacobians for the objective and constraint functions are computed using an automatic differentiation tool called ADOL-C [3]. ADOL-C keeps a record of the elementary computations made during the evaluation of the objective and constraints functions at a given point and reviews this information to produce the corresponding derivatives. Therefore, Jacobian recording (or taping) is performed for 
a new set of objective and constraint functions at each level of optimization. Typically, function evaluations are found to be very expensive for IMRT problems since re-computations of radiation dose to tissue structures are required for each set of beamlet intensities. This excluded an option of using the Finite Difference method for the Jacobian calculations.

\section{IMRT OPTIMIZATION FOR A BRAIN CASE}

In order to demonstrate the general idea of the Lexicographic method, an IMRT optimization case is shown below. It is a relatively simple brain case which involves only 5 structures with 5 axial beams and $\sim 500$ beamlets. Approximately 53,000 dose-calculation points were distributed throughout the structures. For this example, only dose-volume histogram (DVH)based objectives were used for three target volumes (PTV1, PTV2, PTV3), and the chiasm, and a mean dose objective (in a form of quadratic model) was applied for the normal tissue. The planning objectives are summarized in the following table.

\begin{tabular}{|c|c|}
\hline & Planning Objectives \\
\hline $\mathbf{1}^{\text {st }}$ Level & $\begin{array}{c}\text { Dose to the entire target volumes (PTV1, 2, and 3) should be greater than 86.5, 71, and 61 Gy. } \\
\text { Dose to the entire chiasm should be less than 8 Gy. }\end{array}$ \\
\hline $\mathbf{2}^{\text {nd }}$ Level & $\begin{array}{r}\text { Make dose to the three target volumes as uniform as possible, i.e., } \\
\text { Dose to the entire target volumes (PTV1, 2, and 3) should be less than 93.5, 79, and 69 Gy. }\end{array}$ \\
\hline $\mathbf{3}^{\text {rd }}$ Level & Minimize the mean dose of the normal tissue \\
\hline
\end{tabular}

Figure 1 shows the DVHs prior to optimization where the initial intensity pattern of beamlets was chosen randomly.

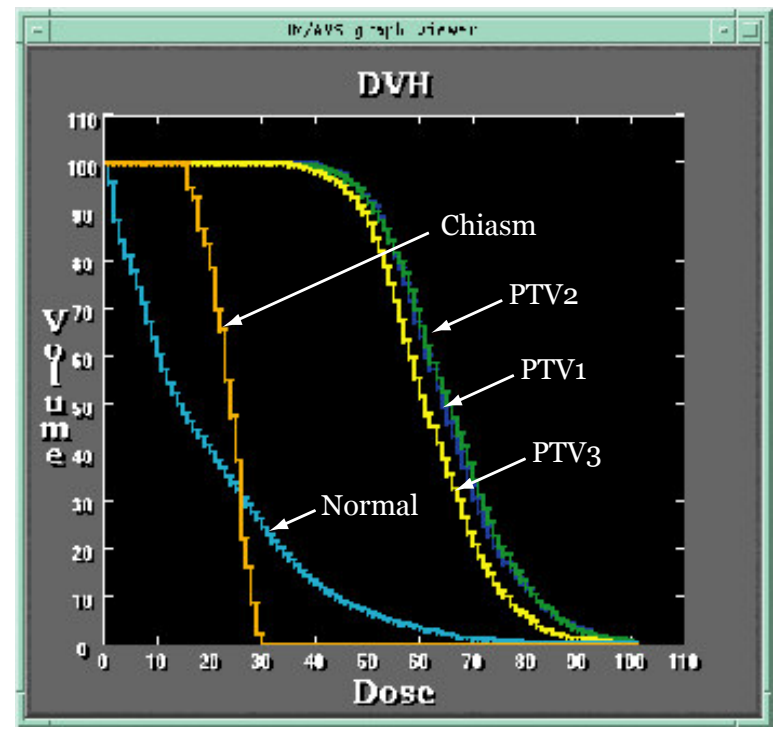

Figure 1. DVH prior to optimization (with randomized beamlet intensities)

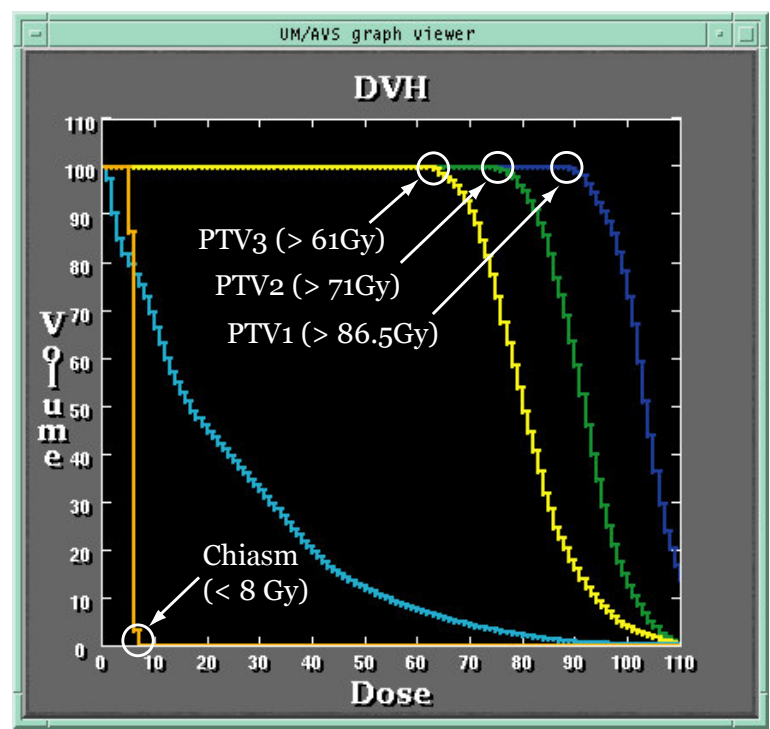

Figure 2. DVH after $1^{\text {st }}$ level optimization

For the $1^{\text {st }}$ level, a total of 4 objectives were identified to have the highest priority. Those are the ones that specify minimization of under-dose for the three PTVs and over-dose for the chiasm. Specifically, the objectives state that $100 \%$ of the volume should receive dose above 86.5, 71, and 61 Gy for PTV1, PTV2, and PTV3, respectively. Zero percent of the volume should be allowed to receive dose above $8 \mathrm{~Gy}$ for the chiasm. These objectives were summed together (with unity weight) and then minimized by the quasi-Newton algorithm (L-BFGS-B). At this level, no constraints other than the lower and upper bounds of beamlet intensity were involved in the optimization. Resulting objective values were all zero indicating the 4 objectives were achieved (shown in Fig. 2). These 4 objectives were then turned into inequality constraints and the next set of objectives was identified for the $2^{\text {nd }}$ level optimization. 


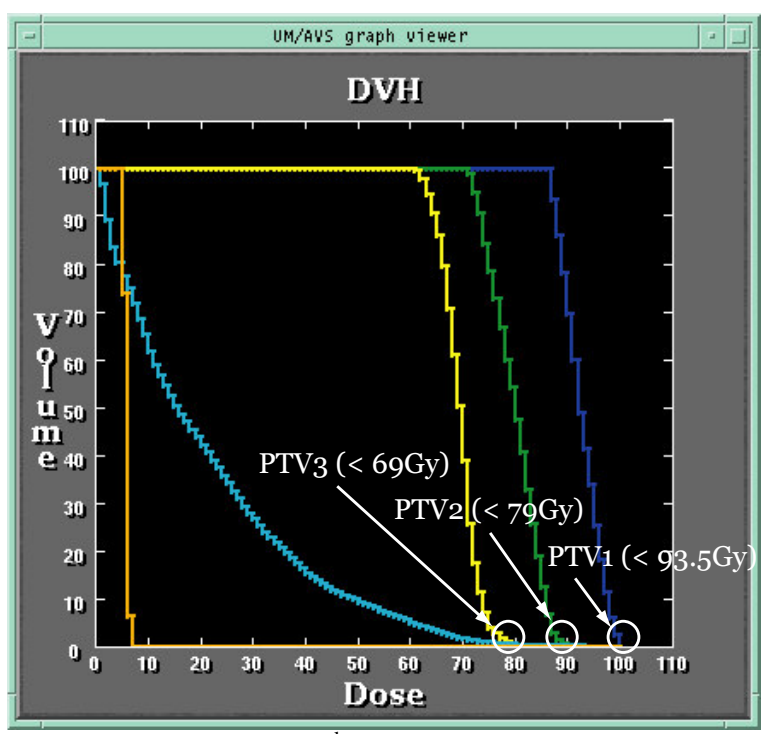

Figure 3. DVH after $2^{\text {nd }}$ level optimization

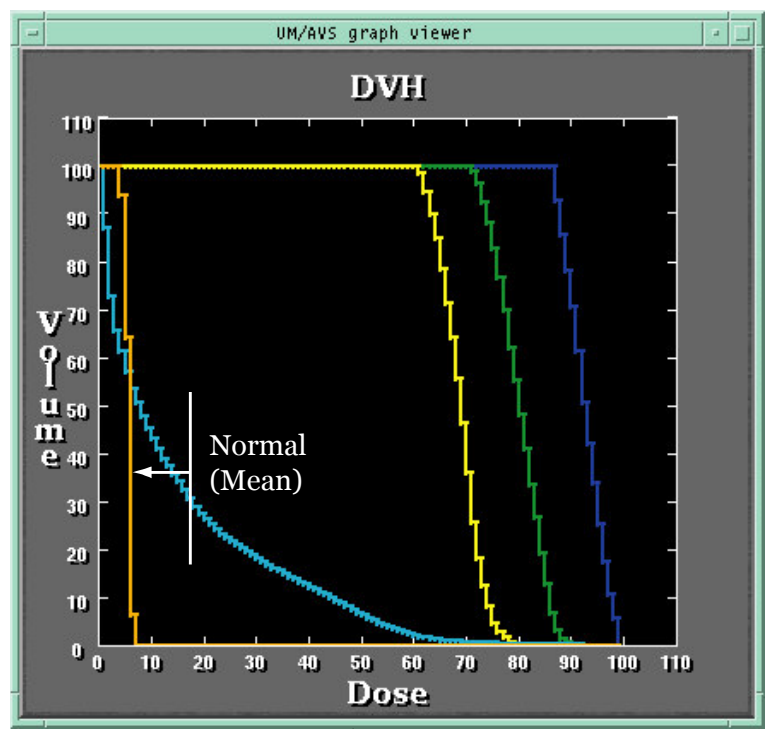

Figure 4. DVH after $3^{\text {rd }}$ level optimization (Final solution)

The $2^{\text {nd }}$ level objectives concern about the uniformity of the target dose distributions. The objectives state that the volumes receiving dose above 93.5, 79, and 69 Gy should be zero percent for PTV1, PTV2, and PTV3, respectively. This non-linear constraint problem was solved by quadratic sequential programming. The resulting objective values were $3.5,11.9$, and 6.1 for PTV1, PTV2, and PTV3, respectively. For the final level, the mean dose of the normal tissue was minimized while keeping all objectives constrained. The optimization resulted in the mean dose of 13.4 Gy as supposed to 20.4 Gy at the $2^{\text {nd }}$ level.

\section{OPTIMIZATION PERFORMANCE}

The following table summarizes the algorithmic performance for the brain case above.

\begin{tabular}{|c|c|c|c|}
\hline & $\mathbf{1}^{\text {st }}$ Level & $\mathbf{2}^{\text {nd }}$ Level & $\mathbf{3}^{\text {rd }}$ Level \\
\hline \# of objective function & 1 & 1 & 1 \\
\hline \# of constraints & 0 & 4 & 7 \\
\hline \# of function calls & 34 & 107 & 468 \\
\hline \# of major iterations & 28 & 55 & 171 \\
\hline
\end{tabular}

\section{CONCLUSION}

The use of $\mathrm{AD}$ tools significantly reduced the development time and efforts for derivative calculations involving a large number of variables in IMRT problems. Early results indicate that highly accurate derivatives are essential to produced both feasible and optimal solutions at a good convergence rate. It was also found that the Lexicographic approach is useful for planners to prioritize planning objectives intuitively and moreover to articulate their priorities into a final solution effectively.

\section{REFERENCES}

1. R. T. Marler, and J. S. Arora, "Review of Multi-Objective Optimization Concepts and Algorithms for Engineering," Technical Report (ODL-01.03), University of Iowa (2003).

2. P. E. Gill, W. Murray, and M. A. Saunders, " SNOPT: An SQP algorithm for large-scale constrained optimization," SIAM Journal on Optimization 12 (2002), 979-1006.

3. A. Griewank and G. F. Corliss, "Differentiation of Algorithms: Theory, Implementation, and Application,” Philadelphia, 1991. SIAM Proceedings of the First International Workshop on Computational Differentiation, January 6-8, (1991). 